\title{
IUCrJ
}

Volume 9 (2022)

Supporting information for article:

findMySequence: a neural-network-based approach for identification of unknown proteins in X-ray crystallography and cryo-EM

Grzegorz Chojnowski, Adam J. Simpkin, Diego A. Leonardo, Wolfram SeifertDavila, Dan E. Vivas-Ruiz, Ronan M. Keegan and Daniel J. Rigden 


\title{
Supplement
}

\section{findMySequence: a neural-network-based approach for identification of unknown proteins in X-ray crystallography and cryo-EM}

\author{
Grzegorz Chojnowski ${ }^{1, \#}$, Adam J. Simpkin ${ }^{2}$, Diego A. Leonardo ${ }^{3}$, Wolfram Seifert-Davila ${ }^{4}$, \\ Dan E. Vivas-Ruiz ${ }^{5}$, Ronan M. Keegan ${ }^{6}$, Daniel J. Rigden²
}

\footnotetext{
${ }^{1}$ European Molecular Biology Laboratory, Hamburg Unit, Notkestrasse 85, 22607 Hamburg, Germany ${ }^{2}$ Institute of Systems, Molecular and Integrative Biology, University of Liverpool, Liverpool L69 7ZB, England

${ }^{3}$ São Carlos Institute of Physics, University of São Paulo, Avenida João Dagnone 1100, São Carlos, SP 13563-120, Brazil

${ }^{4}$ European Molecular biology Laboratory. Meyerhofstraße 1, 69117, Heidelberg, Germany

${ }^{5}$ Laboratorio de Biolgía Molecular, Facultad de Ciencias Biológicas, Universidad Nacional Mayor de San Marcos, Av. Venezuela Cdra 34 S/N, Ciudad Universitaria, Lima, Peru ${ }^{6}$ UKRI-STFC, Rutherford Appleton Laboratory, Research Complex at Harwell, Didcot OX11 OFA, England
}

\# Correspondence: gchojnowski@embl-hamburg.de

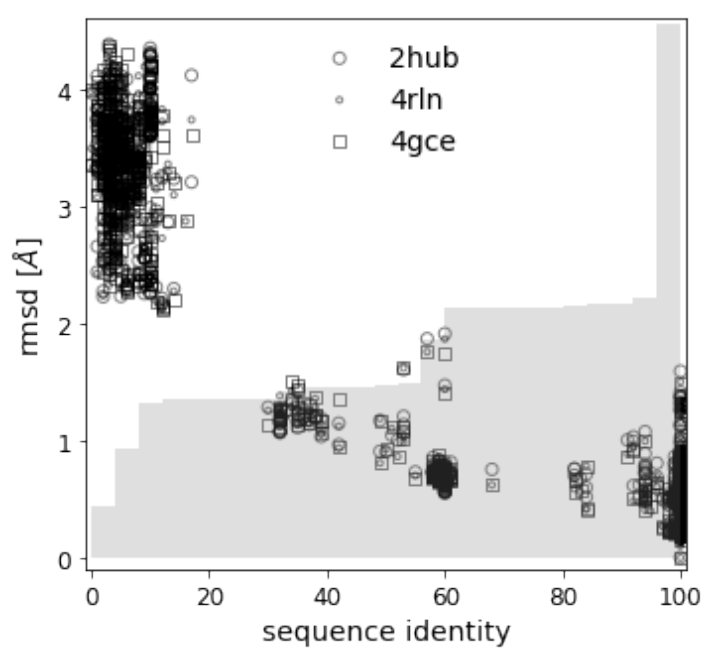

Figure S1 Dependence between CA-atom positions root mean square difference (rmsd) and sequence identity for the hen egg-white lysozyme crystal structure identification benchmark set target structures (PDB id codes 2hub, 4rln, and 4gce) and corresponding search models. Sequence identities and rmsd values were calculated using CLUSTALW2 (Larkin et al., 2007) and GESAMT (Krissinel, 2012) respectively with default parameters. A cumulative histogram of the benchmark set search models to corresponding targets is shown in grey (in arbitrary units). 


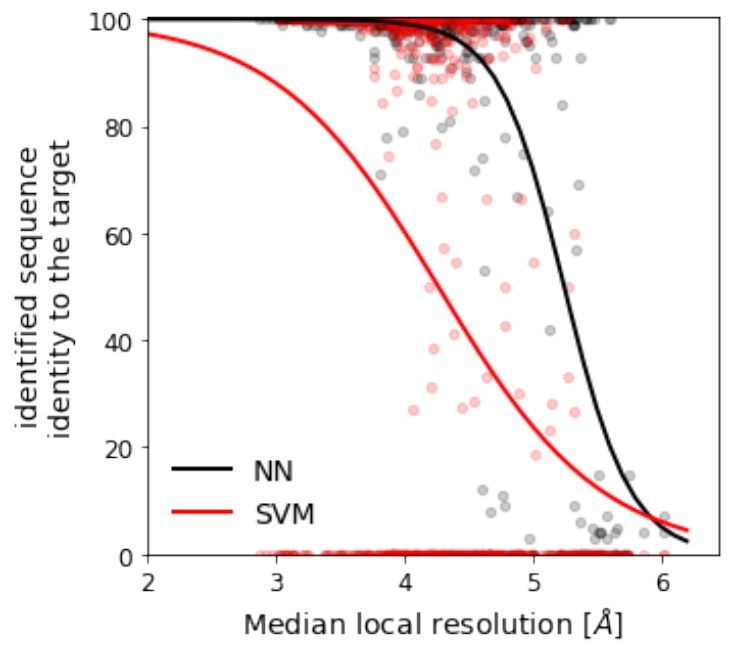

a)

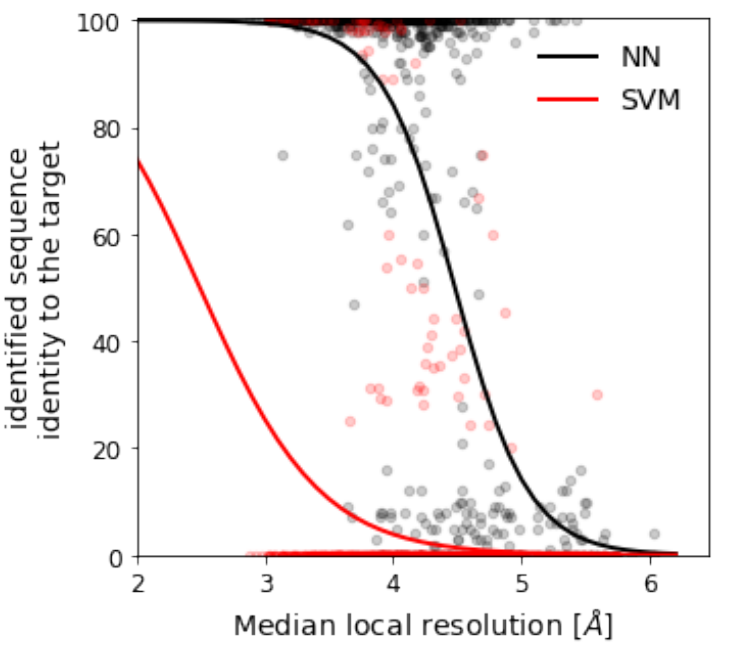

b)

Figure S2 Comparison of sequence identification benchmark results for 909 cryo-EM models of ribosomal proteins and corresponding proteomes for a Neural Network (NN) residue type classifier presented in this work and corresponding Support-Vector Machine (SVM) classifier implemented in ARP/wARP 8.0 (Chojnowski et al., 2019). The two methods were compared for a) refined deposited coordinates and b) models built de novo. The continuous curves are logistic-regression estimates of a probability that an identified sequence will have at least $80 \%$ sequence identity to the target sequence.

\section{References}

Chojnowski, G., Pereira, J. \& Lamzin, V. S. (2019). Acta Crystallogr. Sect. D Struct. Biol. 75, 753-763.

Krissinel, E. (2012). J. Mol. Biochem. 1, 76.

Larkin, M. A., Blackshields, G., Brown, N. P., Chenna, R., McGettigan, P. A., McWilliam, H., Valentin, F., Wallace, I. M., Wilm, A., Lopez, R. \& others (2007). Bioinformatics. 23, 2947-2948. 